\title{
Propagation of spin waves in a 2D vortex network
}

Zhenghua $\mathrm{Li}^{1}$, Bin Dong ${ }^{1 *}$, Yangyang $\mathrm{He}^{1}$, Aiying Chen ${ }^{2}$, Xiang $\mathrm{Li}^{2 *}$, Jing-Hua Tian $^{3}$ Chenglin $\operatorname{Yan}^{3}$

${ }^{1}$ Key Laboratory of New Energy and Rare Earth Resource Utilization of State Ethnic Affairs Commission, School of Physics and Materials Engineering, Dalian Minzu University, Dalian, 116600, China

${ }^{2}$ School of Materials Science and Engineering, University of Shanghai for Science and Technology, Shanghai, 200093, China

${ }^{3}$ College of Energy, Soochow Institute for Energy and Materials InnovationS \& Collaborative Innovation Center of Suzhou Nano Science and Technology, Soochow University, Suzhou 215006, China

*Corresponding authors: dong@dlnu.edu.cn, xiangli@usst.edu.cn 


\section{Section 1: The designed nanostructure}

Fig. $\mathrm{S} 1$ is the designed nanostructure containing four cross-type chiral substructures (marked by Part 1 to Part 4, for chiral control of magnetic vortices), which are featured by the non-vertical cross of $\mathrm{Ni}_{80} \mathrm{Fe}_{20}$ nanostrips and bias nanomagnets (BNs).

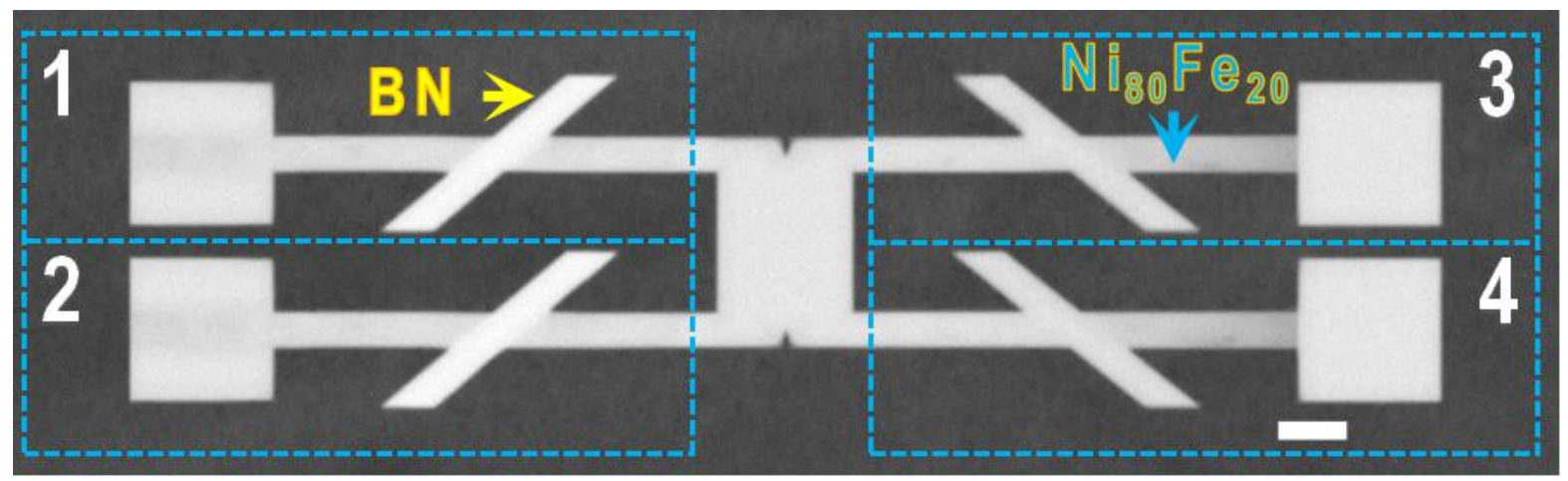

Fig. S1. SEM observation of the nanostructure containing four cross-type chiral substructures. The white color bars represent $1.5 \mu \mathrm{m}$.

\section{Section 2: Functionalities of different parts of the device}

Fig. S2 depicts the SEM observation of the designed device with four square pads (SQs), two $\mathrm{Ni}_{80} \mathrm{Fe}_{20}$ nanostrips, four bias nanomagnets (BNs), two notches and one vortex coupler (VC). The four SQs are responsible for the generation of vortices with $\mathrm{CW}$ or $\mathrm{ACW}$ chirality. The two $\mathrm{Ni}_{80} \mathrm{Fe}_{20}$ nanostrips are used for the injection and transportation of vortices. The four slanted BNs are capable of the chiral control of vortices (See Supporting Information, Section 3). The two notches can facilitate the formation of $\mathrm{CW}-\mathrm{ACW}-\mathrm{CW}$ chains through the generation of the $\mathrm{ACW}$-cores (See Supporting Information, Section 9). The VC are responsible for the hybridization of the two CW-ACW-CW chains. 


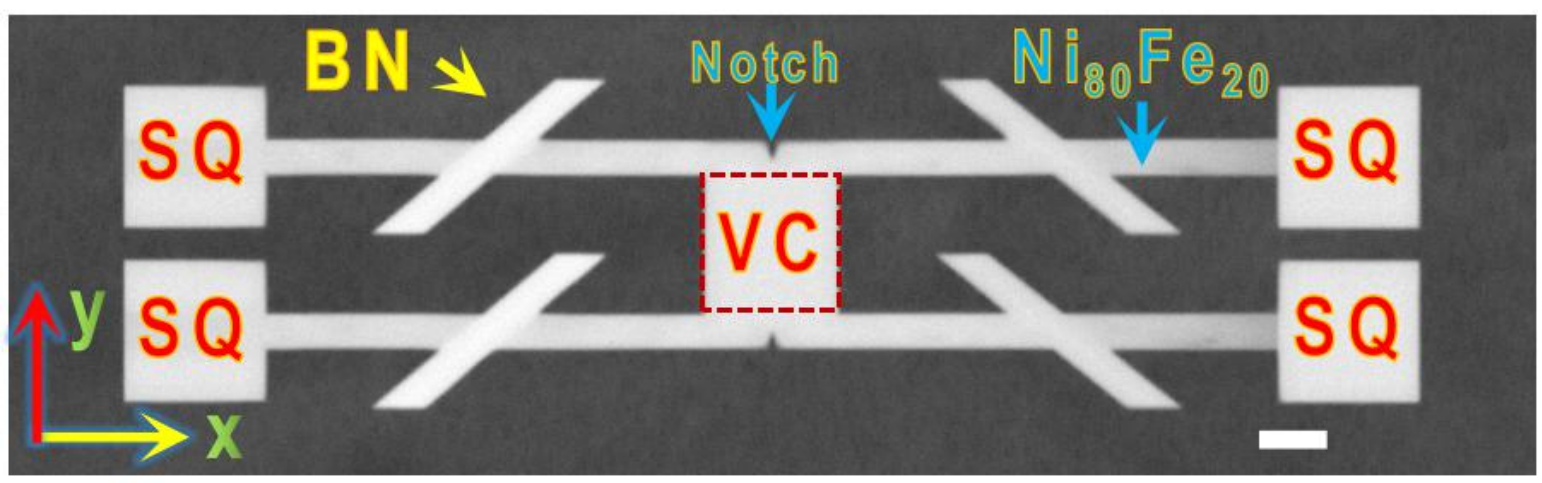

Fig. S2. SEM observation of the device. The white color bars represent $1.5 \mu \mathrm{m}$.

\section{Section 3: Chiral control of magnetic vortex by $B N$}

Fig. S3 depicts the micromagnetic analysis of the BN. The winding number of the system is also shown in Fig. S3. The chirality reversal of a vortex from ACW to $\mathrm{CW}$ is shown for $45^{\circ}$ cross-type nanostructure. The vortex with ACW chirality is injected into the $\mathrm{Ni}_{80} \mathrm{Fe}_{20}$ nanostrip and parked before the $\mathrm{BN}$, as seen in Fig. $\mathrm{S} 3$ (a). The vortex moves towards the BN, and gradually contracts its size, as shown in Fig. S3 (b). The initial ACW core annihilates, while a CW core nucleates near the cross point of the $\mathrm{Ni}_{80} \mathrm{Fe}_{20}$ nanostrip and $\mathrm{BN}$, as seen in Fig. S3 (c). The CW core gradually grows (Fig. S3 (d)) and finally escapes from the BN (Fig. S3 (e)) . Notably, the topological defects of the system are conserved during the process. Fig. S4 depicts the similar process of injecting a $\mathrm{CW}$-vortex. For $45^{\circ}$ cross-type nanostructure, only $\mathrm{CW}$-vortex can be generated when the vortices $(\mathrm{CW}$ and $\mathrm{ACW})$ pass through the $\mathrm{BN}$, proving that the chiral control of vortex can be realized by using this cross-type nanostructure. 

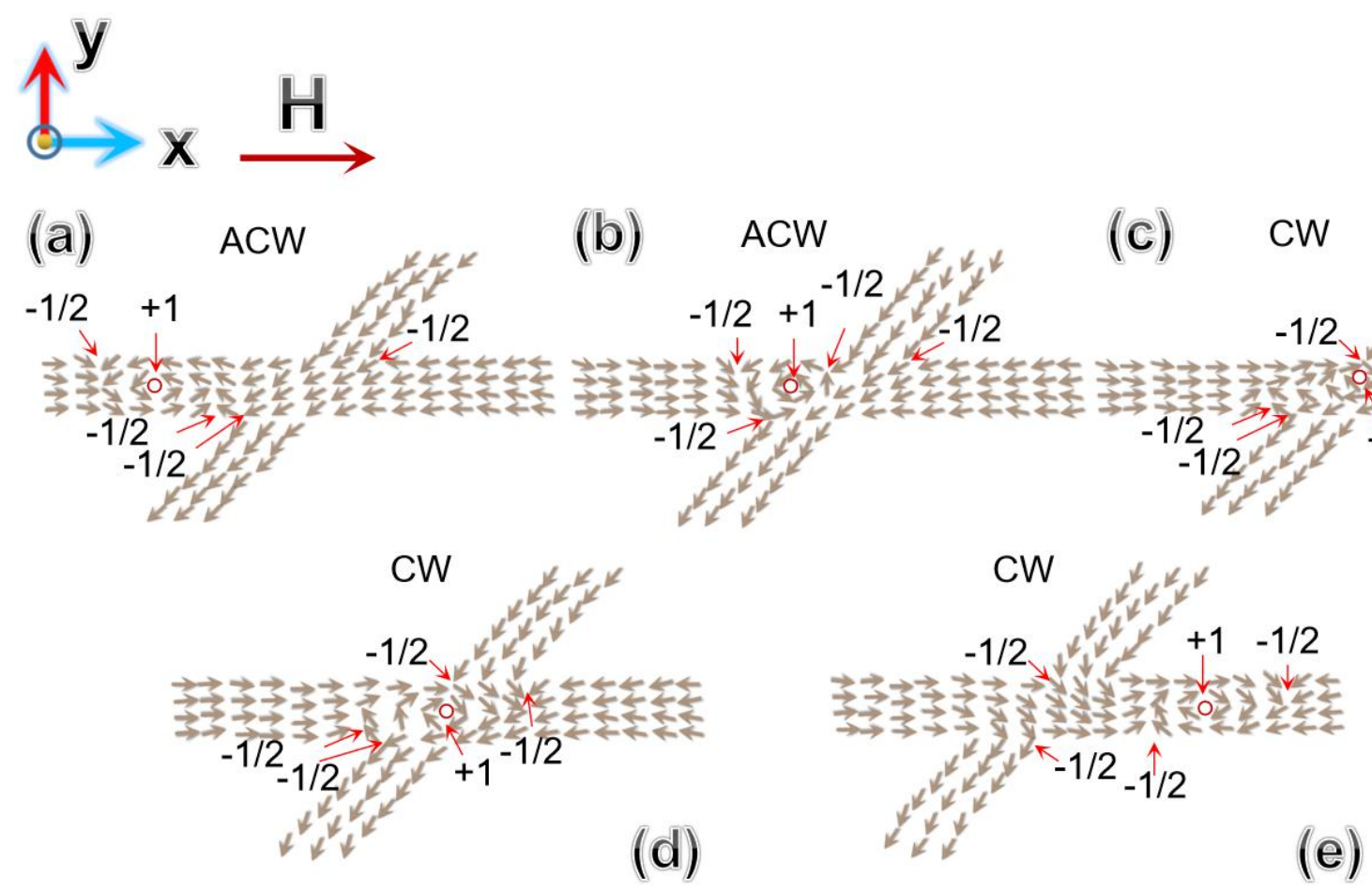

Fig. S3. The chirality reversal of vortex from $\mathrm{ACW}$ to $\mathrm{CW}$, the winding number is marked.

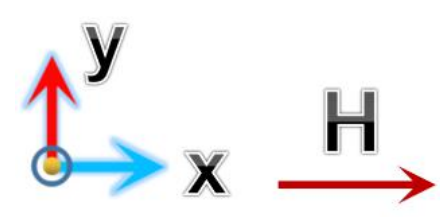

(a) $\mathrm{cW}$

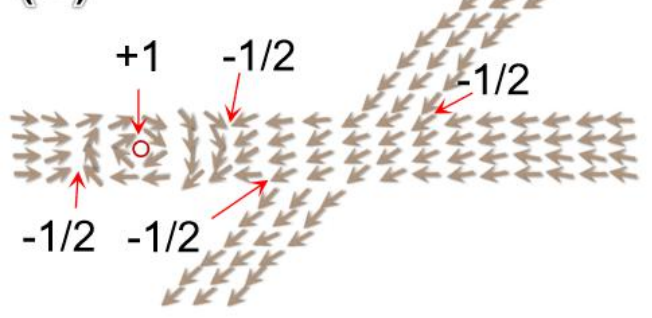

$-1 / 2-1 / 2$

(b) $\mathrm{CW}$

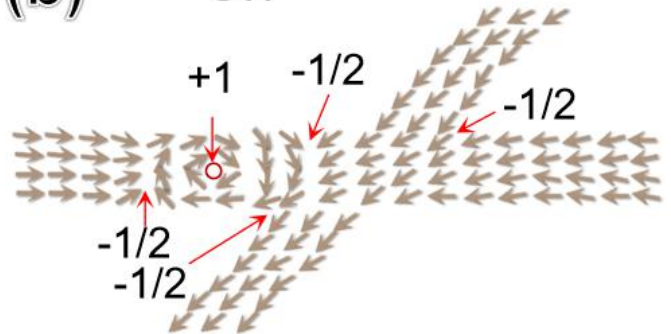

(c)

CW

(d)

CW
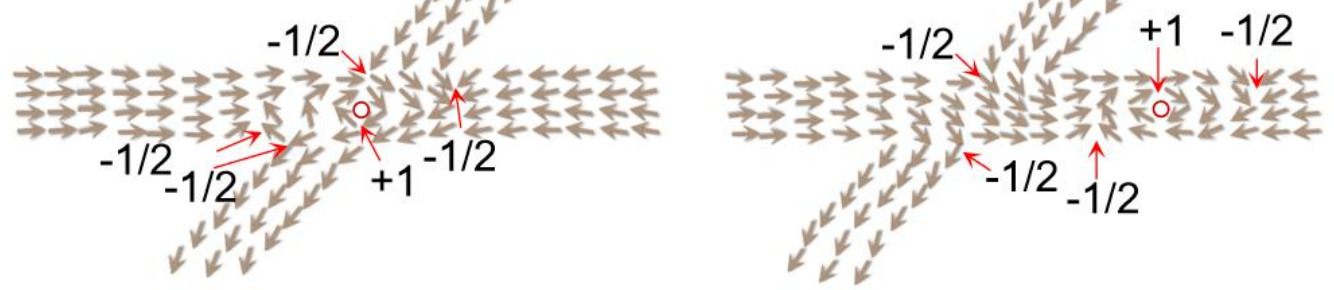

Fig. S4. The process of injecting a CW-vortex, the winding number is marked. 
Fig. S5 shows the AF-MFM images of the cross-type nanostructures with the angle of $30^{\circ}, 45^{\circ}, 60^{\circ}$ and $90^{\circ}$ between the $\mathrm{Ni}_{80} \mathrm{Fe}_{20}$ nanostrips and $\mathrm{BNs}$, respectively. In the case of $30^{\circ}$ and $60^{\circ}$ cross-type nanostructures, the chirality reversal of vortex from $\mathrm{ACW}$ to $\mathrm{CW}$ can be observed, similar to the case of $45^{\circ}$ cross-type. However, the chiral rectifying of vortex can not be achieved with the cross angle of $90^{\circ}$, because the interaction with $\mathrm{BN}$ leads to the transformation of the domain wall from vortex to transverse wall (TW).
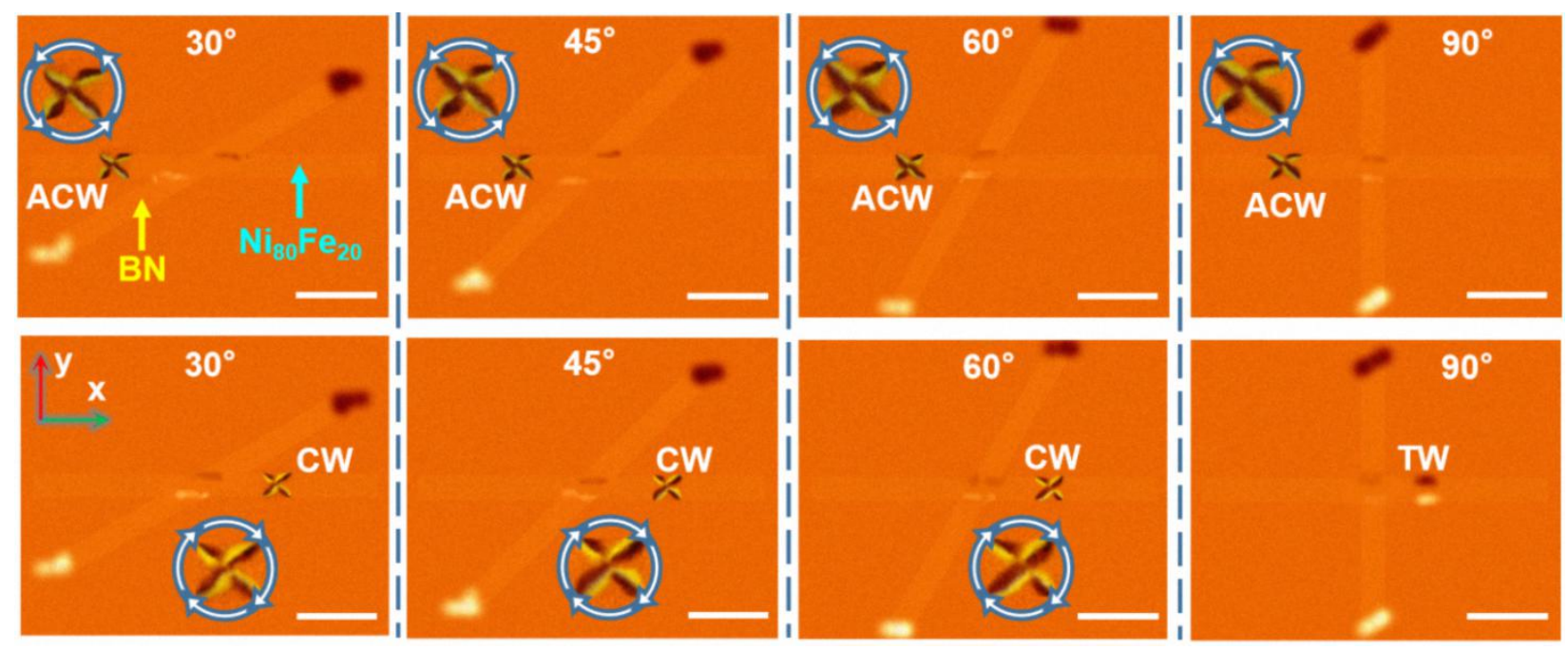

Fig. S5. AF-MFM images of the cross-type nanostructures with the angle of $30^{\circ}, 45^{\circ}$, $60^{\circ}$ and $90^{\circ}$, respectively. The while colour bars represent $1 \mu \mathrm{m}$.

\section{Section 4: Design of the VC size}

The VC is $3 \mu \mathrm{m}$-long and $3 \mu \mathrm{m}$-width (Fig.1 (a), marked in red square box), which is consistent with the length of a CW-ACW-CW chain $(\sim 3 \mu \mathrm{m})$. The injection of a CW-ACW-CW chain can not be achieved with smaller length of VC, as depicted in Fig. S6 (a), the interaction between the vortex chain and device edges deteriorate the chain structure. Contrarily, for VC with longer length than that of vortex chain, the CW-ACW-CW chain can not conserve its original size and structure when injected into the VC, as depicted in Fig. S6 (b), thus, the hybridization of two vortex chains can not be manipulated in a controllable way. 


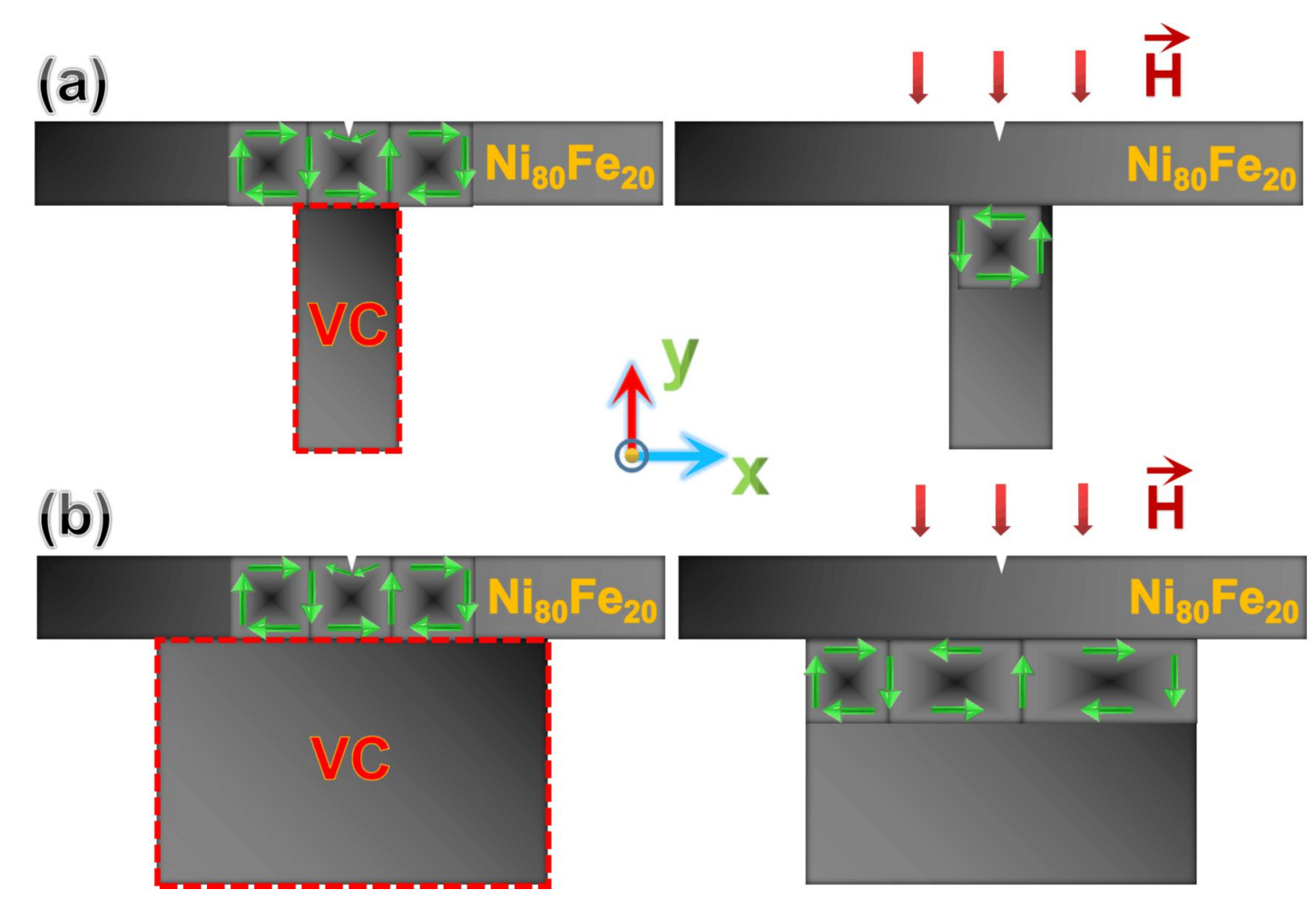

Fig. S6. Schematics of injecting a CW-ACW-CW chain with different size of VC.

\section{Section 5: The formation of higher-order-vortex}

Fig. S7 depicts the schematics of constructing a higher-order-vortex structure. When two vortices (CW and $\mathrm{CW}$ ) approach one another, they interact with each other, and hybridize through the generation of a new vortex core for energy minimization (the mechanism of CW-CW is demonstrated in Fig. S8). Therefore, these two vortices interact and form a triple-coupled-vortex (TCV) structure, including three vortices with one $\mathrm{ACW}$ vortex in the center and two $\mathrm{CW}$ vortices on both sides, as depicted in Fig. S7 (a). Similarly, as two TCV chains (CW-ACW-CW and CW-ACW-CW) converge and interact with each other, these two chains hybridize and produce an additional chain structure (ACW-CW-ACW) for decreasing the magnetic energy. Thus, these two chains converge and generate a $3 \times 3$ matrix of coupled-vortex $(2 \mathrm{D}$ vortex network), with opposite chiralities between the neighbors, as shown in Fig. S7 (b). Based on this principle, it is possible to obtain more complicated coupled-vortex structures, e.g., 1D n-vortex chain $(n \geq 3)^{41}, 2 D m \times n$ vortex network $(m, n \geq 3)$, and so on. 
(a)

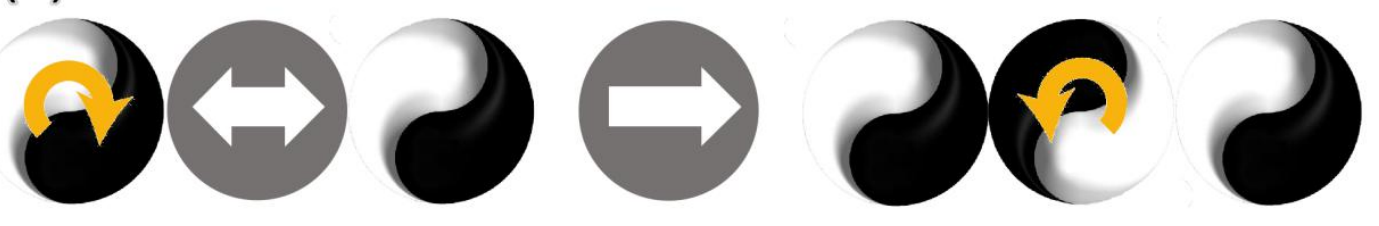

(b)

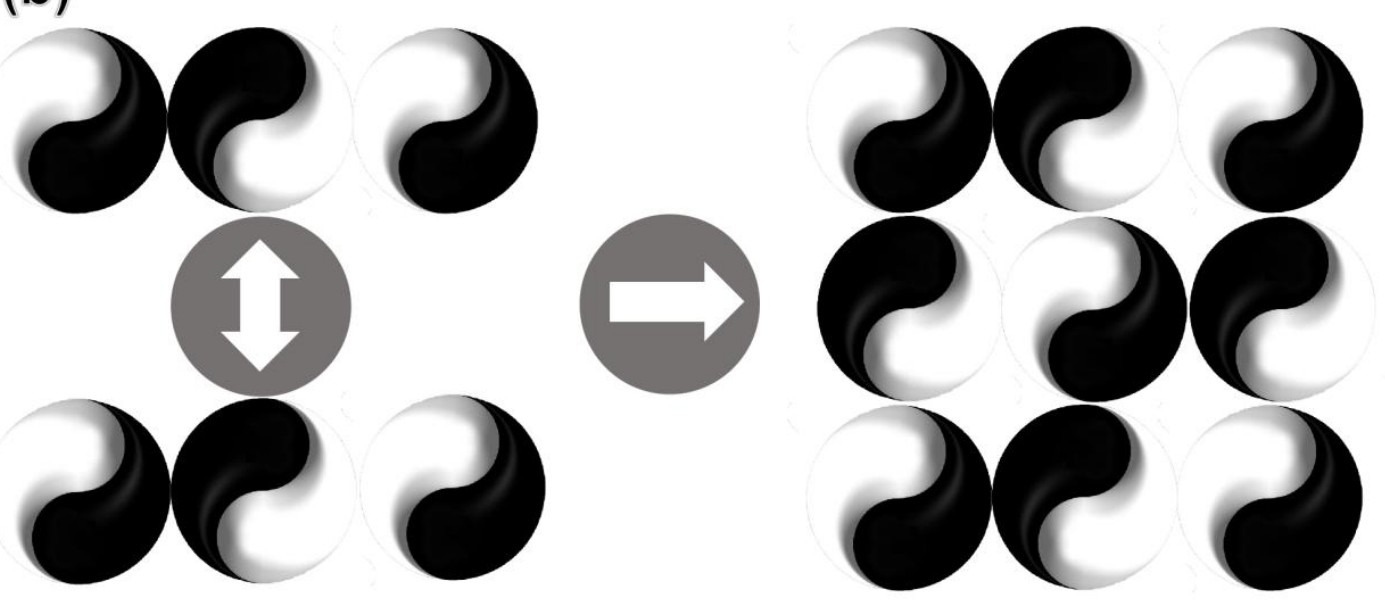

Fig. S7. (a) Two vortices (CW and $\mathrm{CW}$ ) interact with each other to create a triple-coupled-vortex (TCV) structure. (b) Two TCVs approach one another to form a $3 \times 3$ vortex network structure.

Fig. S8 (a)-(d) show the formation of a double-coupled-vortex (DCV) by micromagnetics. The studied model is a $3.15 \mu \mathrm{m}$ long, $300 \mathrm{~nm}$ wide $\mathrm{Ni}_{80} \mathrm{Fe}_{20}$ nanostrip. The nanostrip is initially magnetized by a magnetic field $(\mathrm{H})$ of $1000 \mathrm{Oe}$ along the $-\mathrm{x}$ axis, and then $\mathrm{H}$ along $+\mathrm{x}$ axis is configured as the following sequence: 0 $\mathrm{Oe} \rightarrow 110 \mathrm{Oe} \rightarrow 130 \mathrm{Oe} \rightarrow 150$ Oe. When $\mathrm{H}=0$ Oe, a vortex pair $(\mathrm{CW}$ and $\mathrm{ACW})$ is simultaneously formed inside of the square pads, as shown in Fig. S8 (a). With the increase of $\mathrm{H}$ to $110 \mathrm{Oe}$ along $+\mathrm{x}$ axis, the cores of the vortex pair move from the square pads to the nanostrip, as shown in Fig. S8 (b), suggesting that the magnetic reversal begins by domain wall motion. When $\mathrm{H}$ is further increased to 130 Oe along the $+\mathrm{x}$ axis, the $\mathrm{CW}-\mathrm{ACW}$ vortex pair approaches one another and interacts with each other, it is observed that the vortex pair is weakly coupled through a single-domain (marked by green square) for decreasing the demagnetized energy, as shown in Fig S8 (c). Further increasing $\mathrm{H}$ to $150 \mathrm{Oe}$, the single-domain disappears due to the direct 
exchange and dipolar interaction, and the $\mathrm{CW}-\mathrm{ACW}$ vortex pair directly converges to form a DCV structure (Fig. S8 (d)).

Fig. S8 (e)-(h) show the formation of a triple-coupled-vortex (TCV) using the same field sequence. Fig. S8 (e) and (f) show that vortex pair (CW and $\mathrm{CW}$ ) is simultaneously generated from the two square pads and injected into the nanostrip, similar as the results in Fig. S8 (a) and (b). However, when $\mathrm{H}$ is increased to $130 \mathrm{Oe}$, the $\mathrm{CW}-\mathrm{CW}$ vortex pair is coupled through the generation of a new vortex core at the bottom edge (marked by the red circle) for energy minimization, as shown in Fig. S8 (g). Further increasing $\mathrm{H}$ to $150 \mathrm{Oe}, \mathrm{CW}-\mathrm{CW}$ vortex pair converges to form a TCV (CW-ACW-CW chain), including three vortices with one ACW core in the center and two CW cores on both sides (shown in Fig. S8 (h)). 

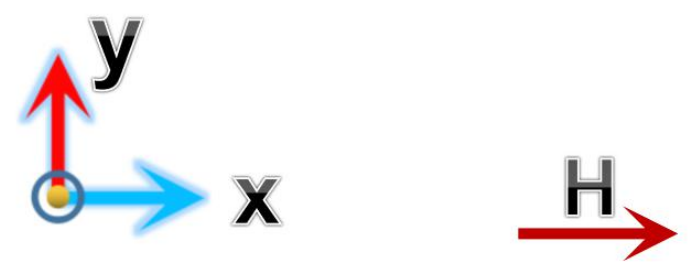

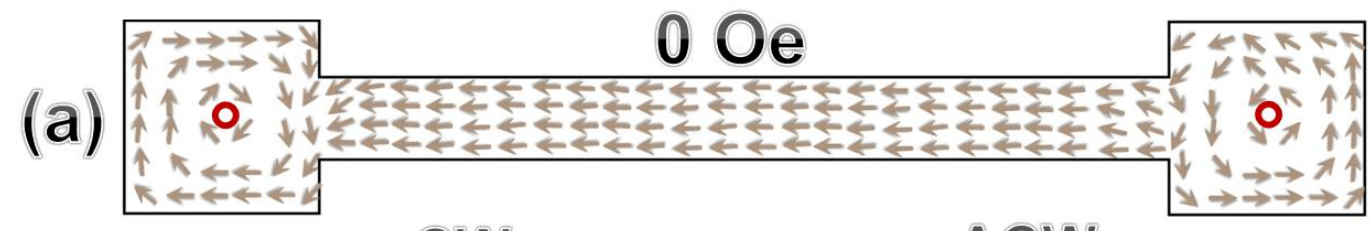

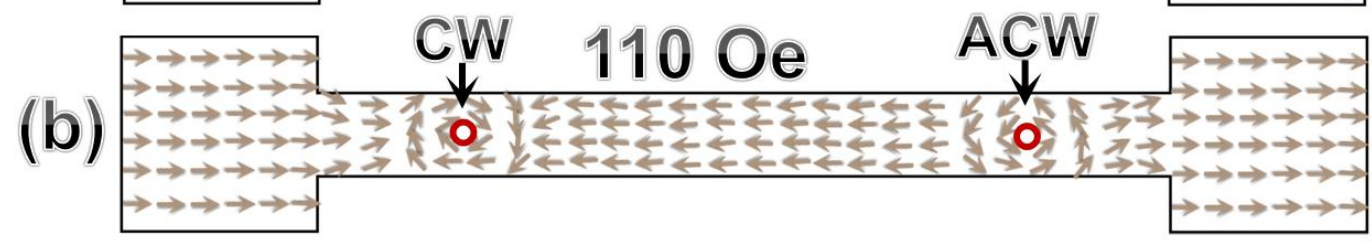

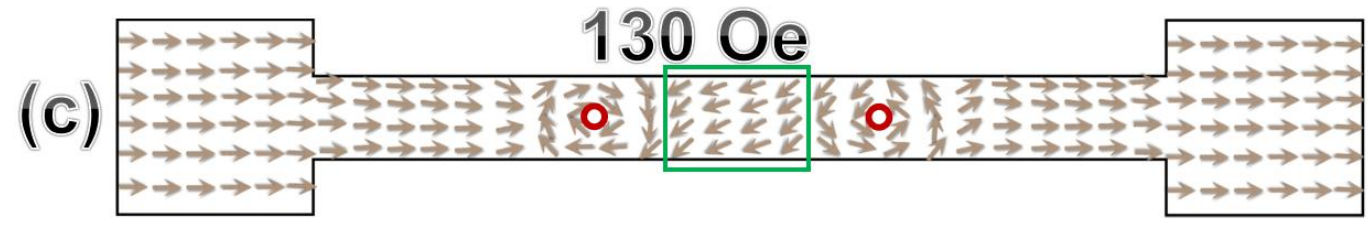

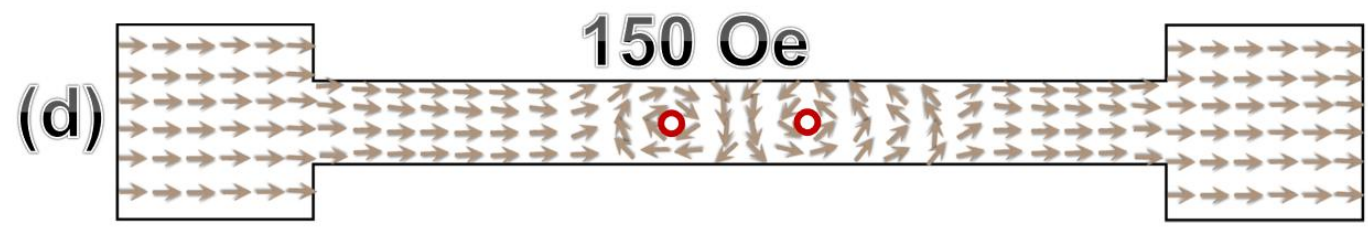

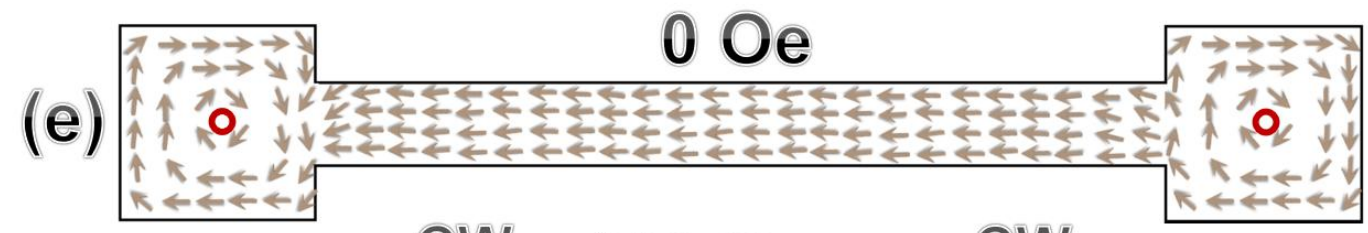

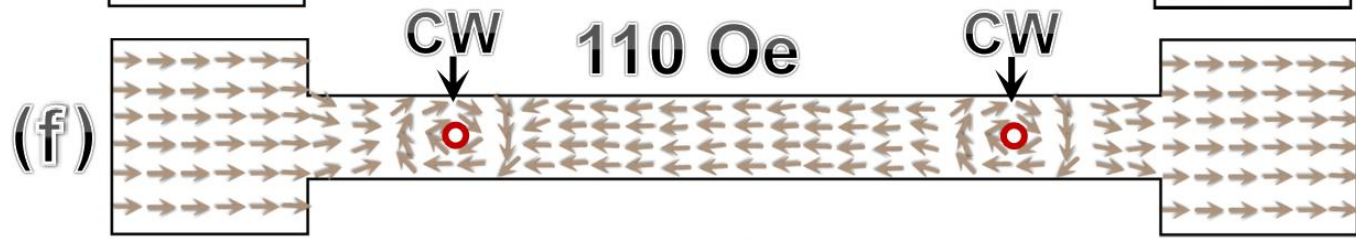

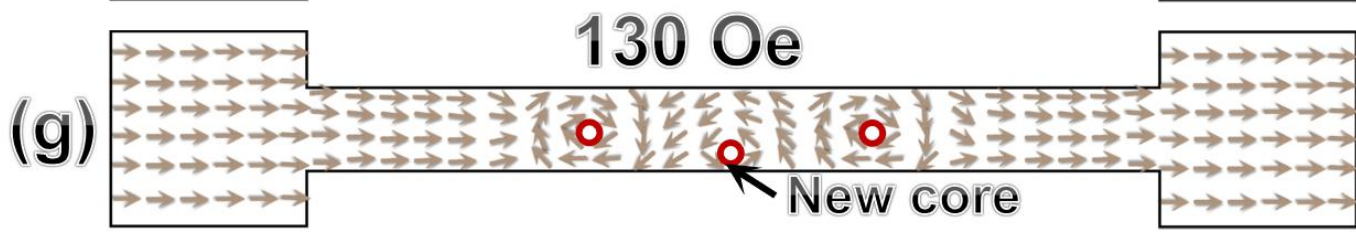

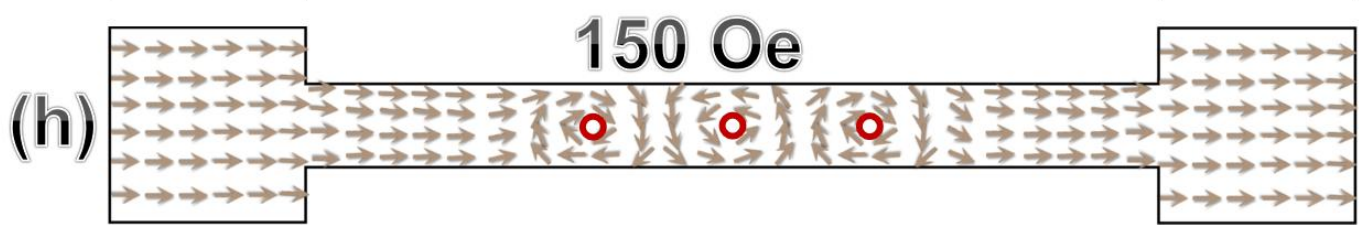

Fig. S8. (a-h) Formation of higher-order-vortex. 


\section{Section 6: Principle of constructing higher-order-vortex}

Fig. S9 (a)-(e) show the schematics of constructing a high-order-vortex structure. Vortex pair (CW and $\mathrm{ACW})$ is simultaneously generated from the square pads and injected into the magnetic nanostrip, as shown in Fig. S9 (a). When the CW-ACW vortex pair interacts with each other, a double-vortex structure can be formed due to the direct exchange and dipolar interaction (Fig. S9 (b)). Here, the CW-vortex is defined as $\mathrm{C}$, and $\mathrm{ACW}$-vortex is $\mathrm{A}$, the formation of a double-vortex is described as:

$$
C \oplus A \rightarrow C A
$$

The CA denote magnetic double-vortex state. According to the equation (S1), a triple-vortex state can also be formed following the same principle:

$$
C \oplus A \oplus C \rightarrow C A C
$$

From the equation (S1), we can deduce the extension equation (S3) of constructing a one dimensional vortex chain by the vortices with different directions of spin circulation, described as:

$$
\underbrace{C_{1} \oplus A_{1} \oplus \cdots \cdots C_{n} \oplus A_{n}}_{2 n} \rightarrow \underbrace{C_{1} A_{1} \cdots \cdots C_{n} A_{n}}_{2 n}
$$

Fig. S9 (c) shows the vortex pair with two CW vortices. The CW-CW vortex pair possesses the same direction of spin circulation, therefore, a new ACW-vortex will be generated when the CW-CW vortex pair interact with each other, as shown in Fig. S9 (d). This process can be described as:

$$
C \otimes C \rightarrow C A C
$$

Here CAC denotes a triple-vortex state. Based on this principle, three CW vortices can form a five-vortex structure, described as:

$$
C \otimes C \otimes C \rightarrow C A C A C
$$

The extension of equation (S5) gives the principle of constructing a one dimensional vortex chain by vortices with the same direction of spin circulation, described as: 


$$
\underbrace{C_{1} \otimes C_{2} \otimes \cdots \cdots \otimes C_{n}}_{n} \rightarrow \underbrace{C_{1} A_{1} C_{2} \cdots \cdots A_{n-1} C_{n}}_{2 n-1}
$$

Fig. S9 (e) shows the schematics of two vortex pairs (two CW vortices at the top and bottom magnetic nanostrips, respectively). When the four CW vortices converge and interact with each other, a $3 \times 3$ vortex network will be formed, as shown in Fig. S9 (f). The formation of a $3 \times 3$ vortex network is defined as:

$$
\left(\begin{array}{lll}
C & \otimes & C \\
\otimes & & \otimes \\
C & \otimes & C
\end{array}\right) \rightarrow\left(\begin{array}{lll}
C & A & C \\
A & C & A \\
C & A & C
\end{array}\right)
$$

According to this principle, a $5 \times 5$ vortex network can be formed as described by the following equation (S8):

$$
\left(\begin{array}{ccccc}
C_{11} & \otimes & C_{12} & \otimes & C_{13} \\
\otimes & & \otimes & & \otimes \\
C_{21} & \otimes & C_{22} & \otimes & C_{23} \\
\otimes & & \otimes & & \otimes \\
C_{31} & \otimes & C_{32} & \otimes & C_{33}
\end{array}\right) \rightarrow\left(\begin{array}{ccccc}
C_{11} & A_{12} & C_{13} & A_{14} & C_{15} \\
A_{21} & C_{22} & A_{23} & C_{24} & A_{25} \\
C_{31} & A_{32} & C_{33} & A_{34} & C_{35} \\
A_{41} & C_{42} & A_{43} & C_{44} & A_{45} \\
C_{51} & A_{52} & C_{53} & A_{54} & C_{55}
\end{array}\right)
$$



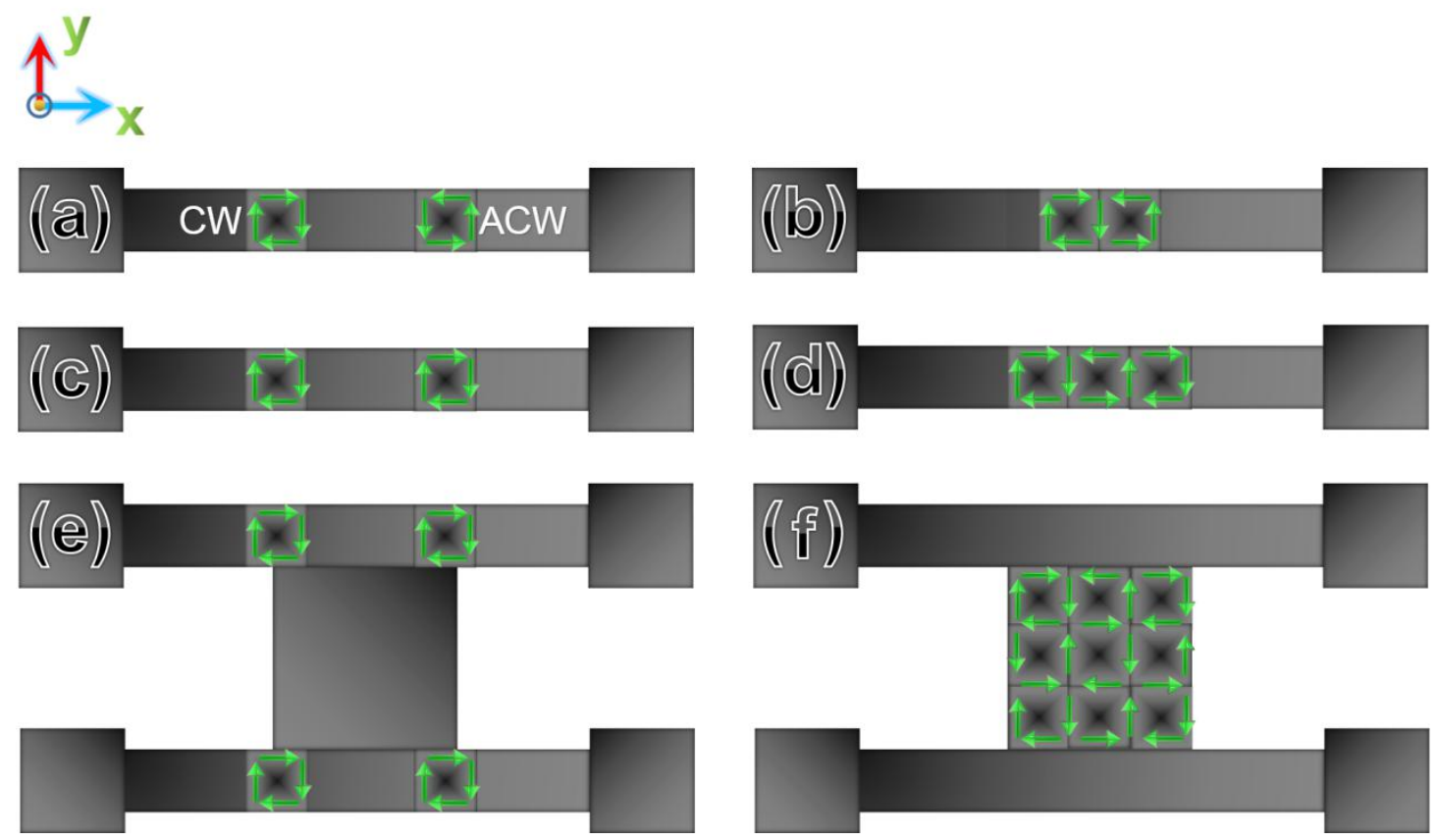

Fig. S9. Schematics of a vortex pair with $\mathrm{CW}$ and $\mathrm{ACW}$ (a) to form a double-vortex structure (b), CW and CW (c) to form a triple-vortex structure (d), two vortex pairs with $\mathrm{CW}$ chirality (e) to form a $3 \times 3$ vortex network structure (f).

\section{Section 7: The analysis of the winding number in a CW-ACW-CW chain}

Here, we prove that the reaction of $\mathrm{CW}+\mathrm{CW}=\mathrm{CW}+\mathrm{ACW}+\mathrm{CW}$ obeys the conservation of topological defects, as shown in Fig. S10. The analysis of the winding number of the system is also presented. The vortex pair with $\mathrm{CW}$ chirality is injected into $\mathrm{Ni}_{80} \mathrm{Fe}_{20}$ nanostrip, as seen in Fig. S10 (a). When two vortices (CW and $\mathrm{CW}$ ) approach one another, they interact with each other, and hybridize through the generation of an ACW-vortex for energy minimization. Finally, a CW-ACW-CW vortex chain forms in the $\mathrm{Ni}_{80} \mathrm{Fe}_{20}$ nanostrip (Fig. $\mathrm{S} 10$ (b)). Notably, the reaction of $\mathrm{CW}+\mathrm{CW}=\mathrm{CW}+\mathrm{ACW}+\mathrm{CW}$ occurs in a confined $\mathrm{Ni}_{80} \mathrm{Fe}_{20}$ nanostrip, the winding number can be created at the sample boundaries, therefore, the topological defects of the system is also conserved during the reaction process. 


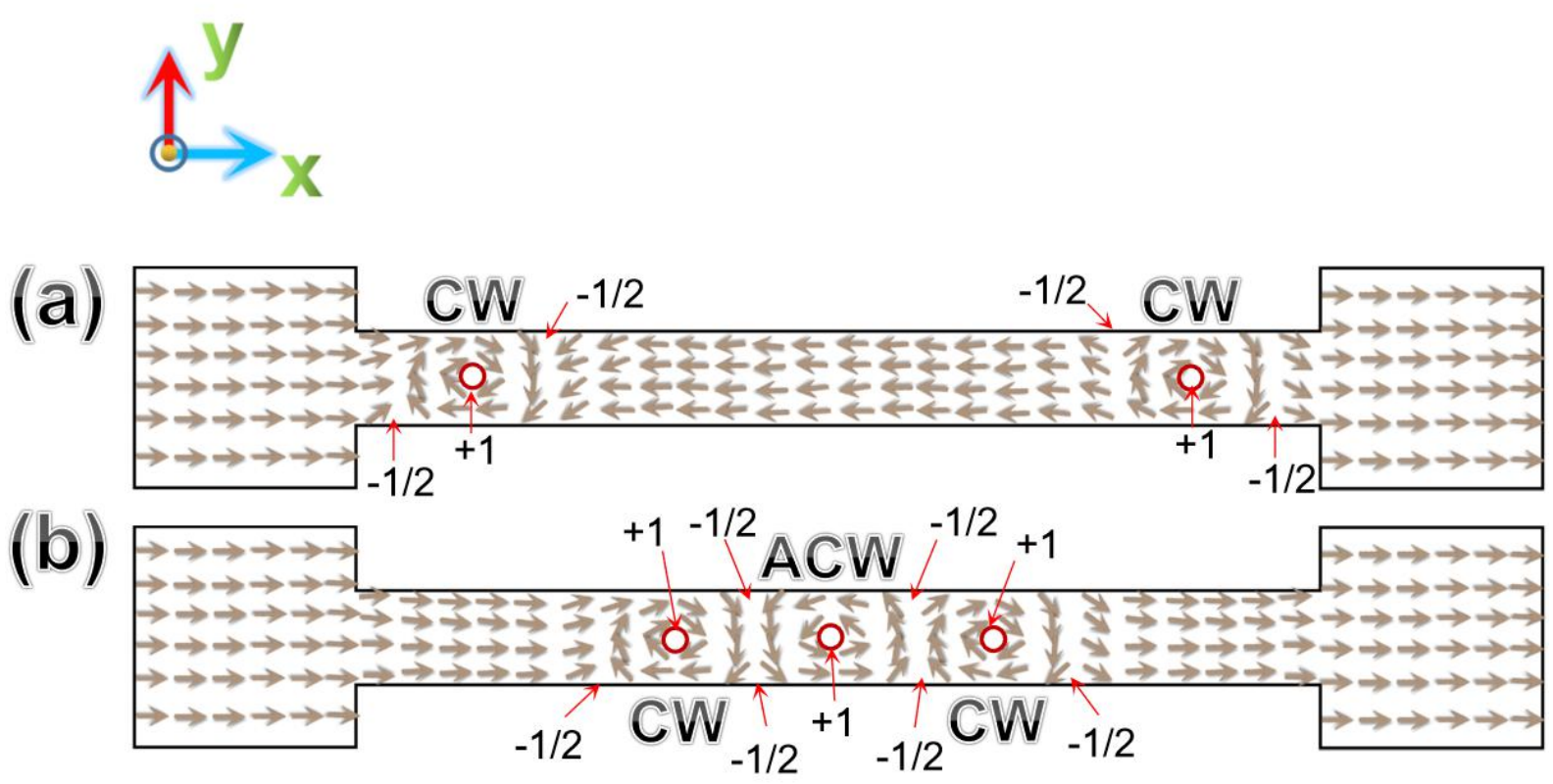

Fig. S10. The formation of a $\mathrm{CW}-\mathrm{ACW}-\mathrm{CW}$ chain from a $\mathrm{CW}$ vortex pair, the winding number is marked.

\section{Section 8: Construction of a 1D vortex chain}

Fig. S11 (a) shows the SEM image of the designed nanostructure with a couple of bias nanomagnets (BNs) and square pads. The slanted BNs are effective for the chiral control of vortices (See Supporting Information, Section 3). The cross angles of these two nanocross are set to be $45^{\circ}$ and $135^{\circ}$, respectively. A notch is introduced at the center of the $\mathrm{Ni}_{80} \mathrm{Fe}_{20}$ nanostrip. The $\mathrm{Ni}_{80} \mathrm{Fe}_{20}$ nanostrip is full saturated via a magnetic field $(\mathrm{H})$ of 1000 Oe along the $-\mathrm{x}$ axis for $5 \mathrm{~s}$, and then $\mathrm{H}$ field along $+\mathrm{x}$ axis is configured as the following sequence: 100 Oe $\rightarrow 150$ Oe $\rightarrow 40$ Oe. MFM images and simulated results of the magnetic configuration of the nanostructure are presented in Fig. S11 (b)-(e). When a DC magnetic field $\mathrm{H}$ of 100 Oe is applied along the $+\mathrm{x}$ axis during the measurements, a vortex pair ( $\mathrm{ACW}$ and $\mathrm{CW}$ ) is injected into the $\mathrm{Ni}_{80} \mathrm{Fe}_{20}$ nanostrip, as shown in Fig. S11 (b). Upon increasing H to 150 Oe along the $+x$ axis for 5 seconds, the vortex pair passes through BNs, as depicted in Fig. S11 (c). Only the right vortex (i.e., $\mathrm{CW}$ ) conserves its chirality, while the left one (i.e., ACW) reverses to $\mathrm{CW}$ after passing through the $\mathrm{BN}$. Upon gradually increasing $\mathrm{H}$ to $40 \mathrm{Oe}$ along the $+x$ axis during the MFM tests, the CW-CW vortiex pair interacts with each 
other and converges to form a CW-ACW-CW vortex chain (Supplement materials, Section 5-6), as seen in Fig. S11 (d). Based on the micromagnetic simulation, the $\mathrm{CW}-\mathrm{CW}$ vortex pair converges and forms a TCV structure (CW-ACW-CW chain), including three vortices with one ACW core in the center and two $\mathrm{CW}$ cores on both sides (shown in Fig. S11 (e)), which can be observed as an stable topological structure in magnetic thin films.

Fig. S11 (f)-(j) show the condition of the device including two same cross angle of $45^{\circ}$. Fig. S11 (g) shows that vortex pair (CW and ACW) are simultaneously generated from the two square pads and injected into $\mathrm{Ni}_{80} \mathrm{Fe}_{20}$ nanostrip, similar as the result in Fig. S11 (b). However, both vortices reverse their chiralities after they pass through BNs, as shown in Fig. S11 (h). The CW-ACW vortex pair interacts with each other via magnetic exchange and dipolar interaction, and converges to form a DCV structure (CW-ACW chain), as shown in Fig. S11 (i). Fig. S11 (j) presents the confirmation of a DCV structure in $\mathrm{Ni}_{80} \mathrm{Fe}_{20}$ nanostrip.
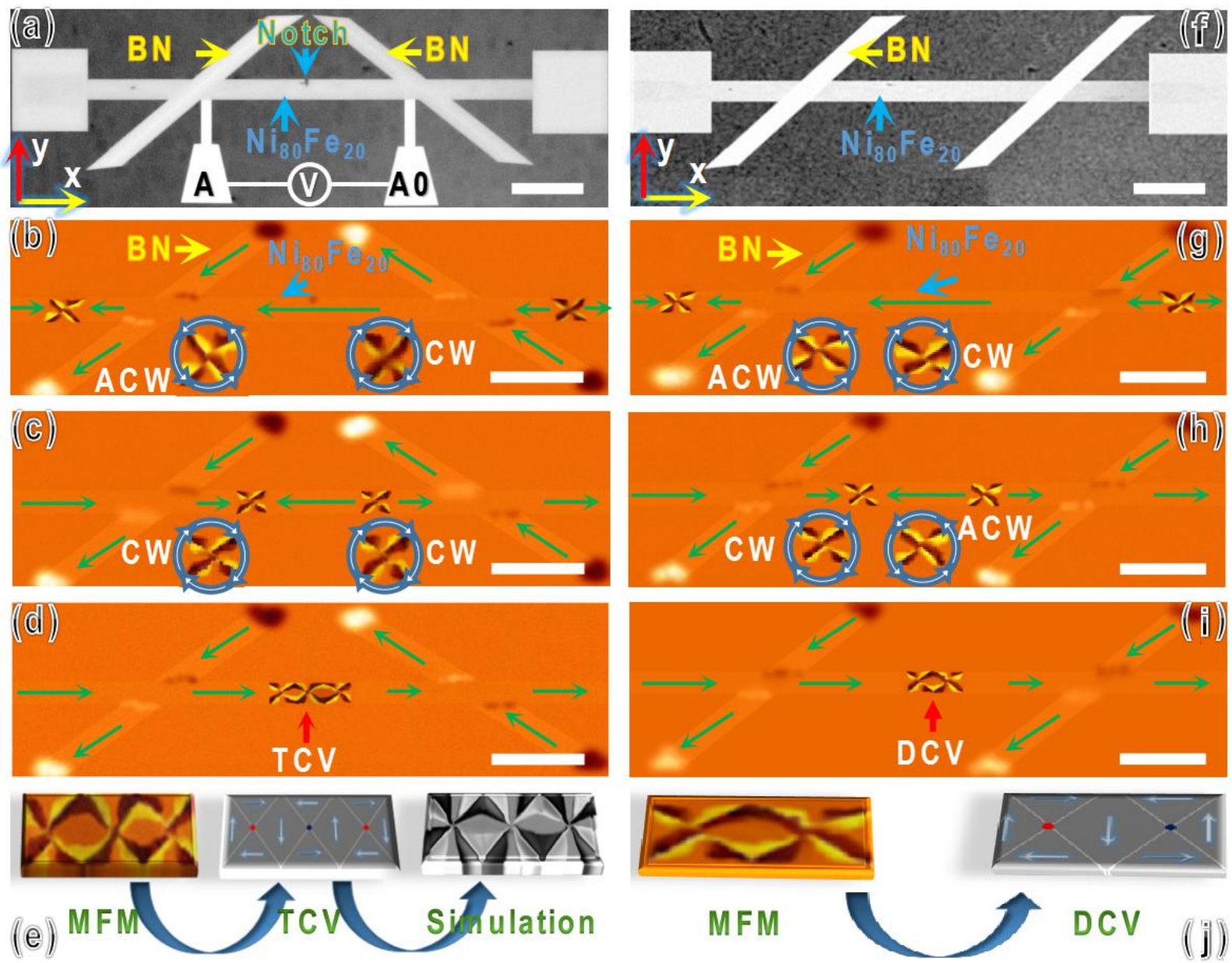
Fig. S11. Construction of a vortex chain. (a) SEM of the $\mathrm{Ni}_{80} \mathrm{Fe}_{20}$ nanostrip and BNs with angle of $45^{\circ}$ and $135^{\circ}$. (b) MFM of vortex pair (CW and ACW) injected into $\mathrm{Ni}_{80} \mathrm{Fe}_{20}$ nanostrips. (c) Vortex pair (CW and CW) passed through BNs. (d) CW-CW vortex pair converges and forms a triple-coupled-vortex (TCV). (e) TCV structure with one ACW core in the center and two CW cores on both sides. (f) SEM of $\mathrm{Ni}_{80} \mathrm{Fe}_{20}$ nanostrip and BNs with the angle of $45^{\circ}$. (g) Vortex pair (CW and ACW) injected into the $\mathrm{Ni}_{80} \mathrm{Fe}_{20}$ nanostrip. (h) Vortex pair (CW and $\mathrm{ACW}$ ) passed through BNs. (i) $\mathrm{CW}-\mathrm{ACW}$ vortex pair converges and forms a double-coupled-vortex (DCV). (j) DCV structure with one ACW core and one CW core. The white colour bars represent $3 \mu \mathrm{m}$. The green arrows represent the magnetization direction.

\section{Section 9: Analysis of the notch on the $\mathrm{Ni}_{80} \mathrm{Fe}_{20}$ nanostrip}

The analysis of the notch on the bottom $\mathrm{Ni}_{80} \mathrm{Fe}_{20}$ nanostrip of the device is depicted in Fig. S12. The studied model is a $3.15 \mu \mathrm{m}$ long, $300 \mathrm{~nm}$ wide $\mathrm{Ni}_{80} \mathrm{Fe}_{20}$ nanostrip integrated with a notch. The vortex pair with $\mathrm{CW}$ chirality is injected into the $\mathrm{Ni}_{80} \mathrm{Fe}_{20}$ nanostrip and parks before the notch, as seen in Fig. S12 (a). When the vortex pair approaches one another, an ACW core and an antivortex core are simultaneously generated near the notch, as seen in Fig. S12 (b). The ACW core grows and finally a CW-ACW-CW chain forms at the notch (Fig. S12 (c)). Notably, the notch of $\mathrm{Ni}_{80} \mathrm{Fe}_{20}$ nanostrip can facilitate the formation of a CW-ACW-CW chain through the generation of an ACW-core. Fig. S13 depict the similar case of the top $\mathrm{Ni}_{80} \mathrm{Fe}_{20}$ nanostrip. 

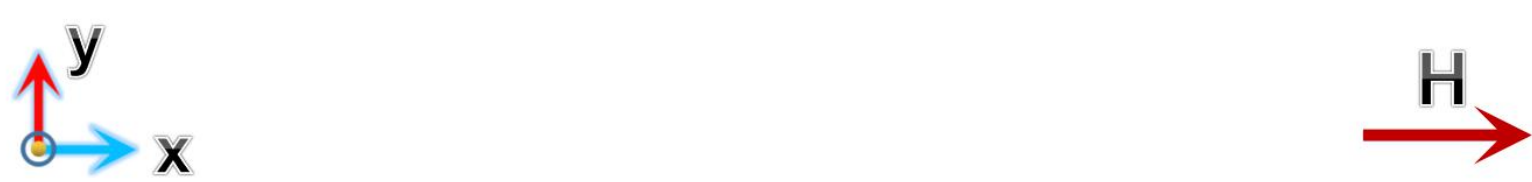

(a)

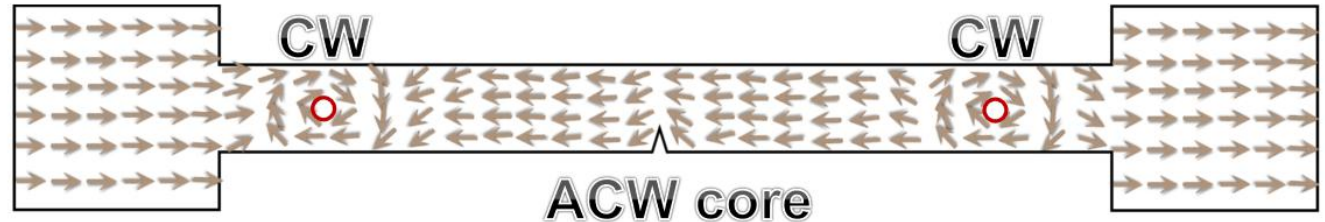

$110 \mathrm{Oe}$

(b)

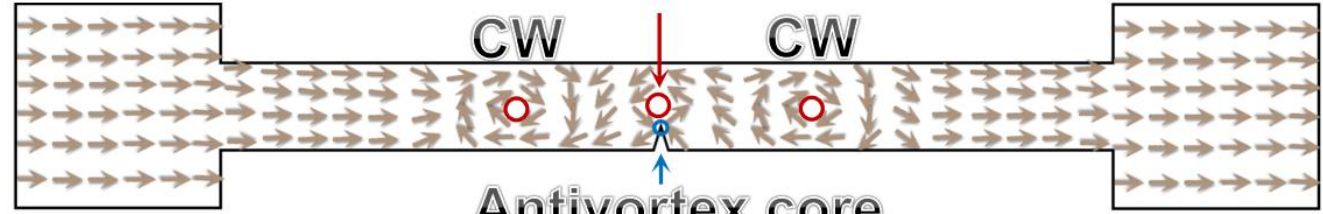

$130 \mathrm{Oe}$

(c)

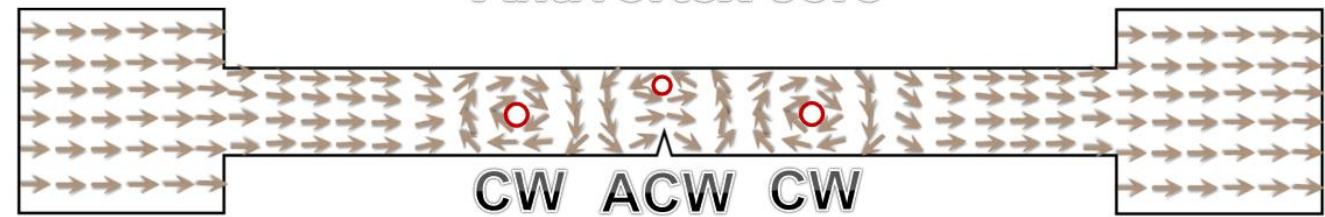

$150 \mathrm{Oe}$

Fig. S12. Analysis of the notch on the bottom $\mathrm{Ni}_{80} \mathrm{Fe}_{20}$ nanostrip of the device.
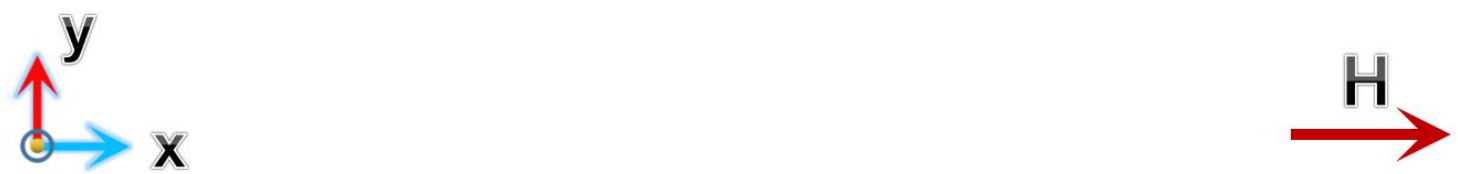

(a)

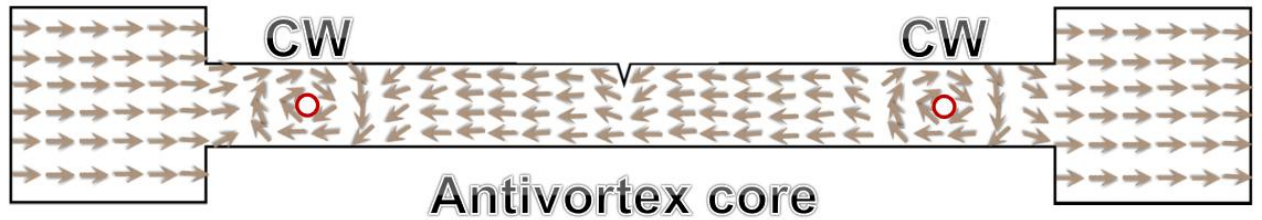

$110 \mathrm{Oe}$

(b)

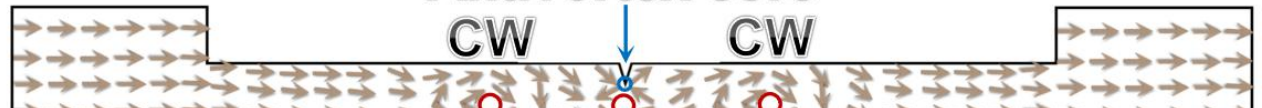

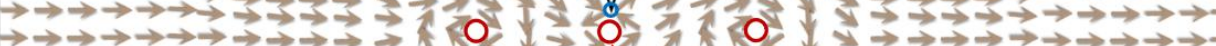

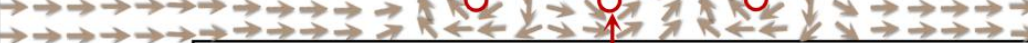

$\vec{b} \rightarrow \rightarrow \rightarrow \rightarrow \rightarrow+\infty$

$130 \mathrm{Oe}$

(c) $\rightarrow \rightarrow \rightarrow \rightarrow-1$ ACW core $\rightarrow \rightarrow \rightarrow \rightarrow \rightarrow$

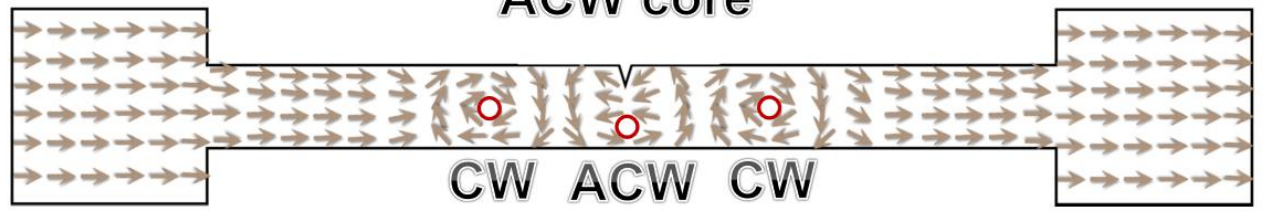

$150 \mathrm{Oe}$

Fig. S13. Analysis of the notch on the top $\mathrm{Ni}_{80} \mathrm{Fe}_{20}$ nanostrip of the device.

Fig. S14 depicts the schematics of injecting a CW-ACW-CW chain from the $\mathrm{Ni}_{80} \mathrm{Fe}_{20}$ nanostrip to the vortex coupler (VC). The CW-ACW-CW chain is formed in 
the $\mathrm{Ni}_{80} \mathrm{Fe}_{20}$ nanostrip and parked at notch, as seen in Fig. S14 (a). The vortex chain moves apart from the notch and is injected into the $\mathrm{VC}$, no extra antivortex core is generated during the process (Fig. S14 (b)). When further increasing the driving magnetic field, the vortex chain will move forward and finally hybridize with another CW-ACW-CW chain. Thus, the notch can not facilitate the injection of $\mathrm{CW}-\mathrm{ACW}-\mathrm{CW}$ chain from the nanostrip into the $\mathrm{VC}$, since no extra antivortex core is produced near the notch during this process.

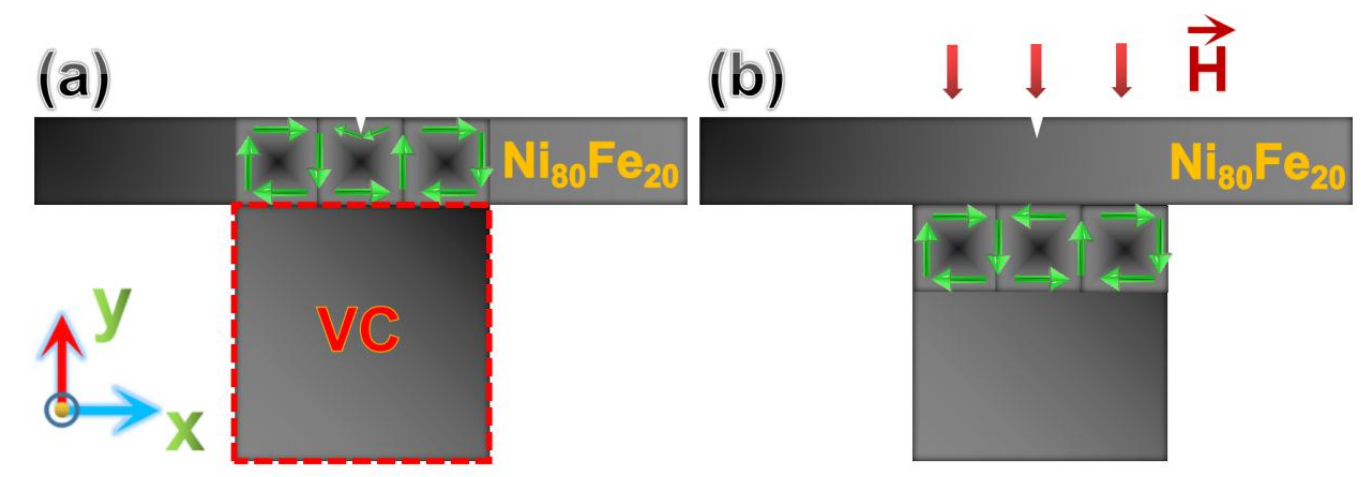

Fig. S14. Schematics of injecting a CW-ACW-CW chain from the $\mathrm{Ni}_{80} \mathrm{Fe}_{20}$ nanostrip into the VC.

\section{Section 10: Quasi-static reversal of vortex network}

The quasi-static magnetization reversal of the vortex network is studied by MFM, as shown in Fig. S15 (a)-(d). The vortex network is induced by a H-field (Hz) perpendicular to the sample surface, and $\mathrm{Hz}$ is configured as the following sequence: $0 \mathrm{Oe} \rightarrow 130$ Oe $\rightarrow 0$ Oe $\rightarrow-130$ Oe. Fig. S15 (a) and (b) show the snapshots of the vortex network in the first reversal process ( 0 to $130 \mathrm{Oe}, \mathrm{Hz}$ along $+\mathrm{z}$ axis), during this stage, the perpendicular component of magnetization obviously enhances, as compared with Fig. S15 (a) and (b). When $\mathrm{Hz}$ reduces from 130 Oe to 0 Oe, the magnetization of vortices returns back to in-plane while keeping a stable network structure (Fig. S15 (c)). Afterwords, the magnetization gradually reverses to $-\mathrm{z}$ axis as $\mathrm{Hz}$ changes from 0 Oe to -130 Oe (Fig. S15 (d)). The quasi-static reversal of vortex network indicates that the vortex network is quasi-stable and can be easily controlled, which opens up a new perspective in the application of magnetic vortices. 


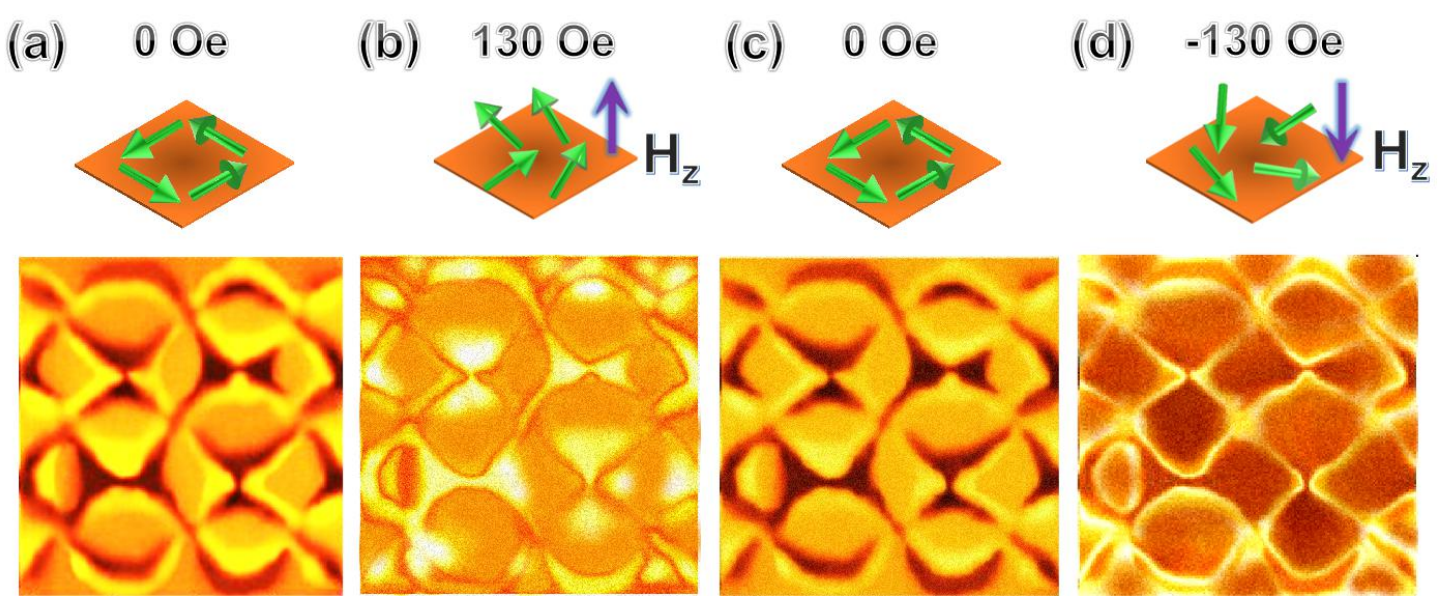

Fig. S15. Quasi-static mechanism of vortex network. (a-d) The MFM images of the vortex network under the magnetic field.

\section{Section 11: Demonstration of Bloch-Néel mixed-type domain walls}

Fig. S16 (a) and (b) depict the X-ray magnetic circular dichroism (XMCD) observations of one network cell (one magnetic vortex). In Fig. S16 (a), the white-black contrast indicates the in-plane magnetization component oriented parallel to the $\mathrm{x}$-axis (white contrast: along the $+\mathrm{x}$ axis; black contrast: along the $-\mathrm{x}$ axis). From Fig. S16 (a), the magnetic ground state should be a closure vortex configuration containing the Néel-type domain walls inside the structure. In Fig. S16 (b), the contrast represents the out-of-plane magnetization component (white contrast: along the $+\mathrm{z}$ axis; black contrast: along the $-\mathrm{z}$ axis). The white-black dipole contrast at the domain walls indicates the existence of Bloch-type domain walls. Therefore, these domain walls are composed of both Bloch and Néel types. Fig. S16 (c) presents a schematic top view of the network cell, showing the mixed Bloch and Néel components. Fig. S16 (d) depicts a cross section view of the network cell (along the dashed line in Fig. S16 (c)), the Bloch component (out-of-plane magnetization component at the domain wall) should arise from the magnetic dipole interaction of nearest vortices, as depicted in Fig. S16 (d). 

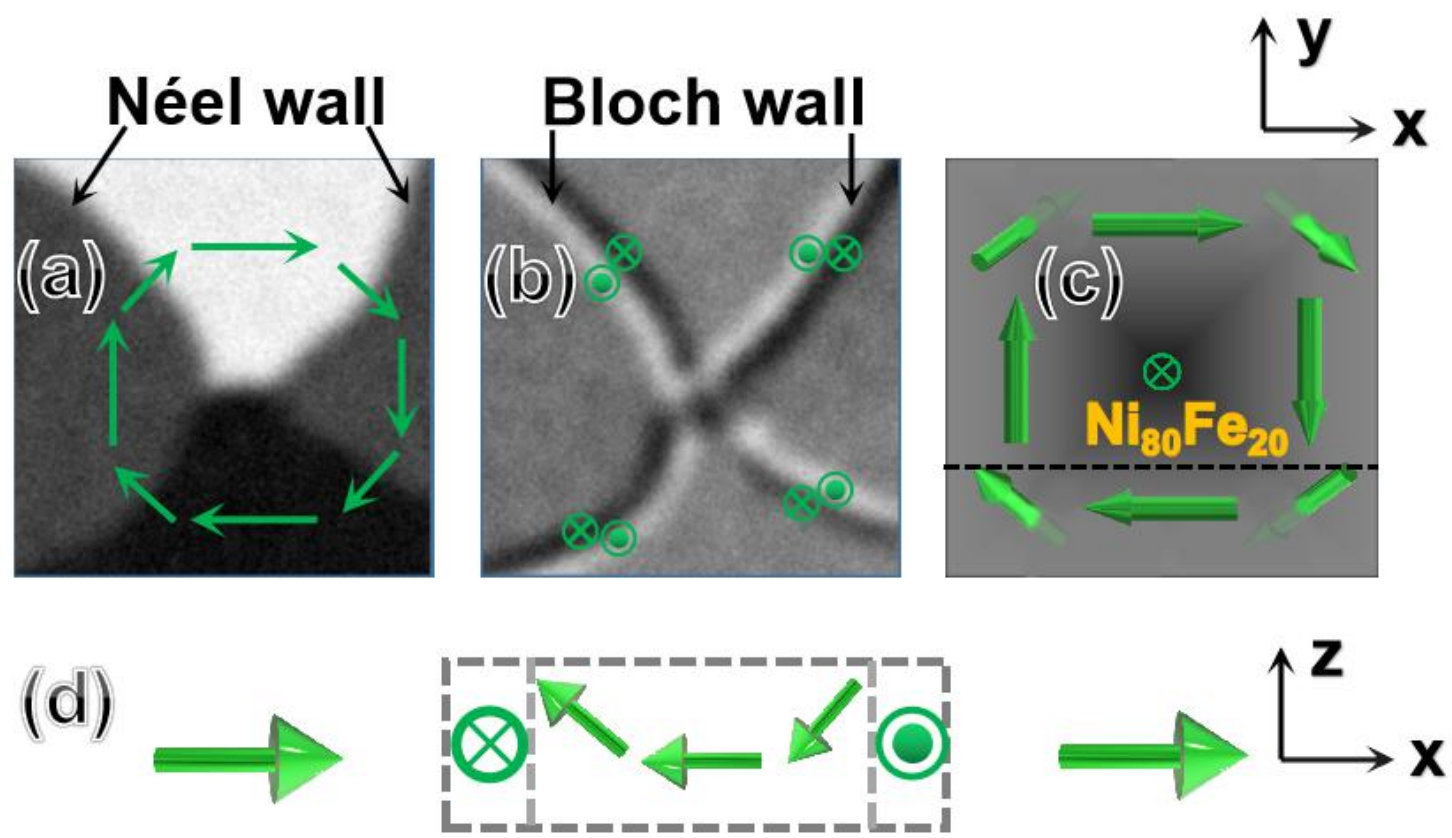

Fig. S16. (a-b) The X-ray magnetic circular dichroism (XMCD) observations of one network cell. (c-d) Schematic top view and cross section view (along the dashed line in (c)) of the network cell. The arrows indicate the magnetization directions.

\section{Section 12: Vortex network for spin-wave propagation}

Fig. S17 (a) and (b) depict the XMCD image (out-of-plane magnetization component) and schematic top view of the network, showing the configuration of the domain walls. Out-of-plane oscillating magnetic field generated by microwave current $\left(\mathrm{I}_{\mathrm{rf}}=\mathrm{I}_{0} \sin (\omega \mathrm{t})\right.$, with amplitudes of $\left.1 \mathrm{~mA}\right)$ is applied for locally exciting the spin-wave at one vortex core. Fig. S17 (c) depicts the measured spin-wave intensity (measured by Brillouin light scattering spectroscopy) along the red dashed line (marked in Fig. S17 (a), across the domain wall) at the frequency of 2.0 and $5.0 \mathrm{GHz}$, respectively. From Fig. S17 (c), the spin-wave is bounded to the domain wall at the frequency of 2.0 GHz. Upon increasing the frequency to $5.0 \mathrm{GHz}$, the spin-wave propagates into the domain. Micromagnetic simulation is performed to reveal the underlay physics of the spin-wave modes. The calculated $\mathrm{z}$-component magnetization $\left(\mathrm{M}_{\mathrm{z}}\right)$ pattern at selected area (red rectangular box in Fig. S17 (b)) of the two spin-wave modes is inserted in Fig. S17 (c). At the frequency of $2.0 \mathrm{GHz}$ (spin wavelength $\sim 200 \mathrm{~nm}$ ), the spin-wave propagates along the domain wall because of the formation of potential 
well at the domain wall. Upon increasing the frequency to $5.0 \mathrm{GHz}$ (spin wavelength $\sim 95 \mathrm{~nm}$ ), the spin-wave spreads from the domain wall into the domain owing to the increased magnetic exchange energy. In our work, the dispersion relation along the domain wall is almost linear below $4.0 \mathrm{GHz}$, because of the confinement of spin-wave at the domain wall under lower frequency (below 4.0 GHz). For frequency higher than $5.0 \mathrm{GHz}$, the dispersion relation of the spin-wave deviate from the linear constraint, due to the radiation of spin-wave from the domain wall into the domain. 
$\stackrel{y}{\longrightarrow} x$

(a) Microwave antenna

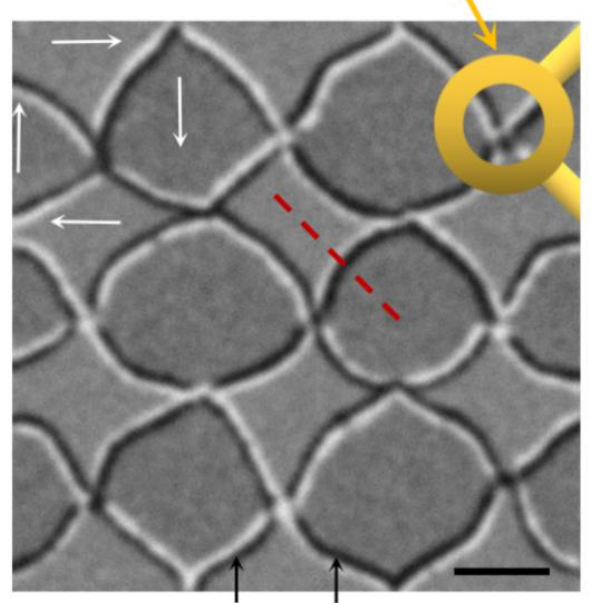

Domain wall
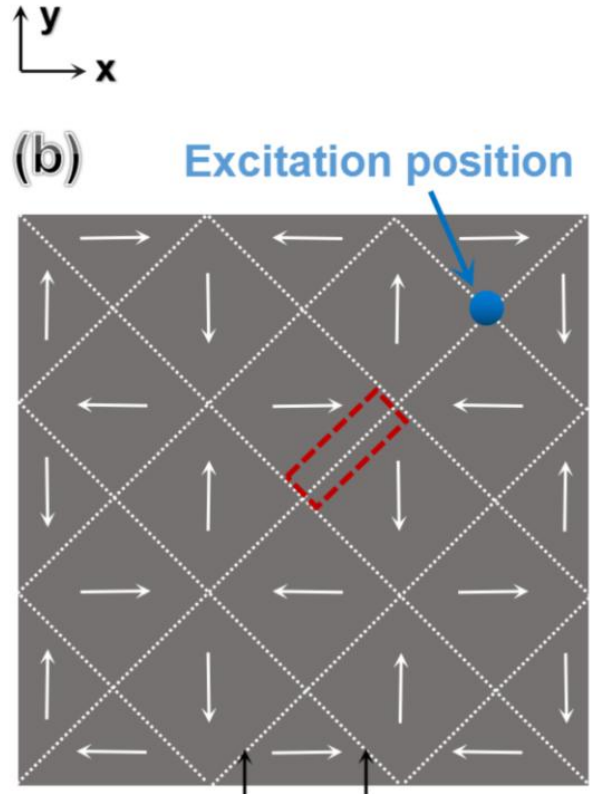

Domain wall

(c)

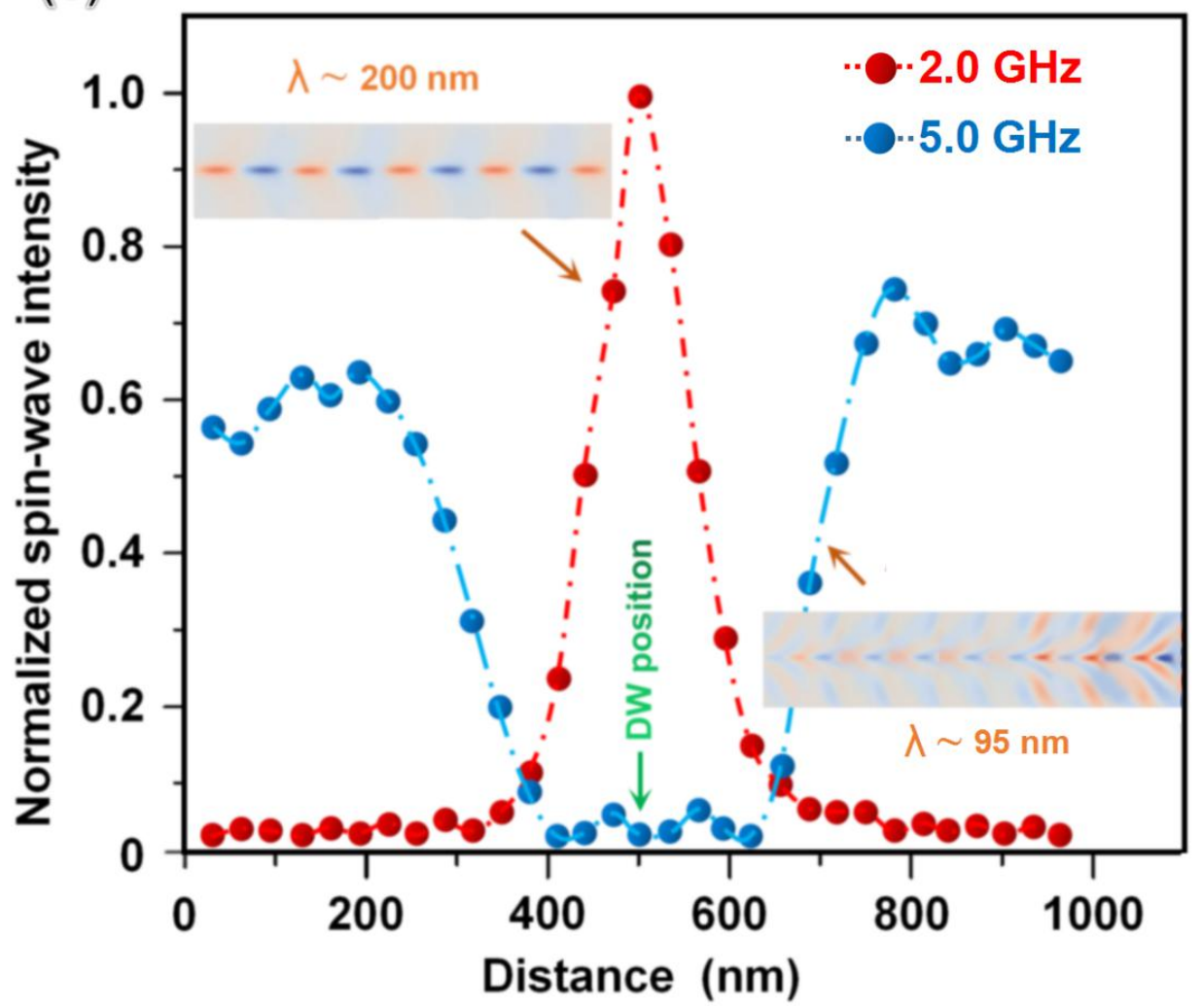

Fig. S17. (a-b) The XMCD image (out-of-plane magnetization component) and schematic top view of the network. (c) The spin-wave intensity measured by Brillouin light scattering spectroscopy along the red dashed line (marked in (a)) at the frequency of 2.0 and $5.0 \mathrm{GHz}$, respectively. The calculated z-component magnetization $\left(\mathrm{M}_{\mathrm{z}}\right)$ pattern at selected area (red rectangular box in (b)) of the two 
spin-wave modes is inserted in (c). The black color bar in (a) represents $0.5 \mu \mathrm{m}$. The white arrows in (a) and (b) represent the magnetization direction.

\section{Section 13: Principles of AF-MFM technique}

Fig. S18 shows the fundamental principles of AF-MFM (Alternating-force magnetic force microscopy) by lock-in technique for detecting sample magnetization. An $\mathrm{AC}$ voltage $\mathrm{V}(\omega)$ is applied to ferrite core to produce a modulated $\mathrm{H}$-field perpendicular to film plane, which can periodically transform magnetization directions in magnetic samples, and generate an $\mathrm{AC}$ magnetic field $\mathrm{B}(\omega)$ from the sample surface. $B(\omega)$ is detected by a hard MFM tip lifted above the sample surface, generating a frequency-modulated MFM signal A $(\omega)$ (frequency modulation of cantilever resonance) which is extracted using a phase-locked-loop. Demodulated signal $\mathrm{C}(\omega)$ is then fed into lock-in amplifier to produce measured MFM signal. In AF-MFM, resonant frequency $\left(\mathrm{f}_{0}\right)$ of cantilever is $256 \mathrm{kHz}$. AC voltage and frequency is $0.2 \mathrm{~V}$ and $100 \mathrm{~Hz}$, respectively. AFM/MFM images are captured under the lift/tapping modes using a high coercivity $\mathrm{L}_{0}$-FePt tip. MFM tip can get closer to material surface without admixing the atomic phase, therefore, spatial resolution of AF-MFM can be better than $5 \mathrm{~nm}$. 


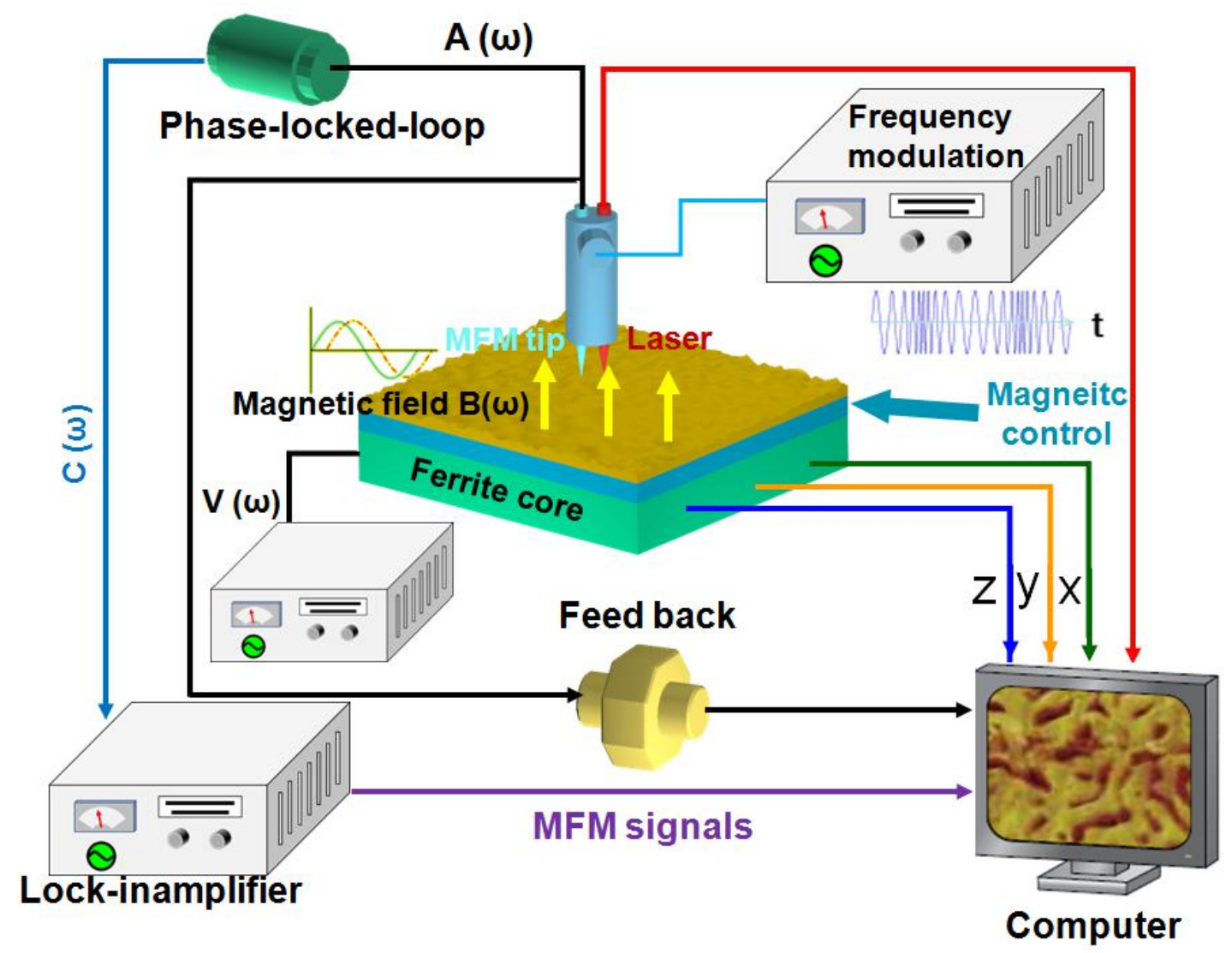

Fig. S18 . Principles of AF-MFM technique.

In AF-MFM, the MFM tip oscillations can be described as

$$
m \frac{d^{2} z(t)}{d t^{2}}+m \gamma \frac{d z(t)}{d t}+\left(k_{0}+\Delta k\right) z(t)=F_{0} \cos \left(\omega_{0} t\right)
$$

here $z, \gamma, \mathrm{m}$ and $k_{0}$ are the displacement, damping constant, effective mass and intrinsic stiffness of the oscillated MFM tip. $\Delta k$ is the effective stiffness factor of the MFM tip, and $F_{0} \cos \left(\omega_{0} t\right)$ is an alternating force from piezo-electric element.

An AC voltage $\mathrm{V}\left(\omega_{\mathrm{m}}\right)$ is applied to the ferrite core to produce a modulated $\mathrm{H}$-field perpendicular to sample surface, which can periodically transform the magnetization directions in magnetic samples. The magnetization $M_{\text {sample }}^{a c}(t)$ of samples rotates periodically,

$$
M_{\text {sample }}^{a c}(t)=M_{z}(t)+M_{x}(t)=M_{z}^{a c} \cos \left(\omega_{m} t\right)+M_{x}^{a c} \sin \left(\omega_{m} t\right)
$$

here $M_{z}^{a c}, M_{x}^{a c}$ are the components of magnetization perpendicular and parallel to the sample surface, respectively. 
The AF-MFM images are imaged under the lift/tapping modes using a high coercivity $\mathrm{L} 1_{0}$-FePt tip (more than $10 \mathrm{KOe}$ ), the MFM tip can be treated as a magnetic monopole, the effective stiffness factor of the MFM tip is defined as

$$
\begin{aligned}
& \Delta k(t) \cong q_{\text {tip }}^{d c} \frac{\partial H_{z}^{a c}\left(M_{\text {sample }}^{a c}(t)\right)}{\partial z} \\
& =q_{t i p}^{d c}\left(\frac{\partial H_{z}^{a c}\left(M_{z}^{a c} \cos \left(\omega_{m} t\right)\right)}{\partial z}+\frac{\partial H_{z}^{a c}\left(M_{x}^{a c} \sin \left(\omega_{m} t\right)\right)}{\partial z}\right) \\
& \cong q_{t i p}^{d c}\left(\frac{\partial H_{z}^{a c}\left(M_{z}^{a c}\right)}{\partial z} \cos \left(\omega_{m} t\right)+\frac{\partial H_{z}^{a c}\left(M_{x}^{a c}\right)}{\partial z} \sin \left(\omega_{m} t\right)\right) \\
& =\Delta k_{0} \cos \left(\omega_{m} t+\phi\right)
\end{aligned}
$$

here $q_{t i p}^{d c}$ is the effective magnetic monopole, $H_{Z}^{a c}$ is the alternating magnetic field from the sample surface, and $\omega_{m}$ is the frequency of AC voltage.

Based on the lock-in technique, the in-phase (X) and out-of-phase (Y) signals, corresponding to the sine and cosine parts in equation (S11), can be extracted as

$$
X+i Y \propto \frac{\partial H_{z}^{a c}\left(M_{z}^{a c}\right)}{\partial z} \cos \left(\omega_{m} t\right)+i \frac{\partial H_{z}^{a c}\left(M_{x}^{a c}\right)}{\partial z} \sin \left(\omega_{m} t\right)
$$

Meanwhile, the amplitude (A) and phase (P) signals can also be extracted by lock-in technique:

$$
\begin{gathered}
A=\left\{\left[\frac{\partial H_{z}^{a c}\left(M_{z}^{a c}\right)}{\partial z}\right]^{2}+\left[\frac{\partial H_{z}^{a c}\left(M_{x}^{a c}\right)}{\partial z}\right]^{2}\right\}^{1 / 2} \\
P=\arctan \left\{\frac{\partial H_{z}^{a c}\left(M_{x}^{a c}\right)}{\partial z} / \frac{\partial H_{z}^{a c}\left(M_{z}^{a c}\right)}{\partial z}\right\}
\end{gathered}
$$

\section{Section 14: Description of the micromagnetic theory}

In the micromagnetic simulation (OOMMF program, code NIST.18), standard parameters of $\mathrm{Ni}_{80} \mathrm{Fe}_{20}$ materials are selected: saturation magnetization of $800 \mathrm{kAm}^{-1}$, crystalline anisotropy constant of $200 \mathrm{~J} / \mathrm{m}^{3}$, exchange stiffness of $7.5 \mathrm{pJm}^{-1}$, and damping coefficient of 0.05 . The energy terms include Zeeman, crystalline anisotropies, shape anisotropies, magnetic exchanges, and magnetostatic energies: 


$$
E_{\text {total }}^{i}=E_{\text {Zee }}^{i}+E_{c k}^{i}++E_{s k}^{i}+E_{e x}^{i}+E_{m}^{i}
$$

The effective field $\vec{H}_{e f f}^{i}$ is defined as :

$$
\vec{H}_{e f f}^{i}=-\frac{1}{\mu_{0}} \frac{\partial E^{i}}{\partial \vec{M}^{i}}
$$

Where $\vec{M}^{i}$ is the magnetization in a grid.

The reversals of magnetic moments obey the law of Landau-Lifshitz-Gilbert (LLG) equations:

$$
\frac{d \vec{M}^{i}}{d t}=-\gamma \vec{M}^{i} \times \vec{H}^{i}{ }_{e f f}-\frac{\alpha}{M_{s}} \vec{M}^{i} \times\left(\vec{M}^{i} \times \vec{H}^{i}{ }_{e f f}\right)
$$

where $M s, \gamma$ and $\alpha$ present the saturation magnetization, gyromagnetic coefficient, and damping constant, respectively. 